\title{
Impact of Galileo data on the solutions of the variometric approach for displacement analysis
}

\author{
Francesca Tesolin \\ University of Trento, via Mesiano 77, 38123 Trento - Italy \\ Alfonso Vitti* \\ University of Trento, via Mesiano 77, 38123 Trento - Italy \\ Augusto Mazzoni \\ University of Rome "La Sapienza ", via Eudossiana 18, 00184 Roma - Italy \\ Mattia Crespi \\ University of Rome "La Sapienza ", via Eudossiana 18, 00184 Roma - Italy
}

\begin{abstract}
The Variometric Approach for Displacement Analysis Standalone Engine (VADASE) was successfully applied for seismological and monitoring purposes using GPS observations. In this work, GPS inter-operable and specific signals transmitted by Galileo satellites were considered to evaluate the impact of Galileo observations on VADASE solutions. The repetition period of the Galileo constellation was investigated to identify the time-windows with the most abundant number of Galileo satellites. Observation packages of about $15 \mathrm{~min}$ at $1 \mathrm{~Hz}$ were analyzed. Comparisons between the GPS derived and the Galileo derived VADASE solutions were performed after the identification of satellite configurations presenting the same number of satellites and similar geometries. Galileo and GPS combined solutions were also investigated. Single frequency and iono-free phase observations from a set of IGS-MGEX sites were processed in order to evaluate the sensitivity of the
\end{abstract}

\footnotetext{
*Corresponding author

Email addresses: francesca.tesolin@alumni.unitn.it (Francesca Tesolin), alfonso.vitti@unitn.it (Alfonso Vitti), augusto.mazzoni@uniroma1.it (Augusto Mazzoni), mattia.crespi@uniroma1.it (Mattia Crespi) 
VADASE solutions with respect to a zero-displacement scenario. Galileo derived solutions are, in general, of good quality, essentially comparable to or better than the GPS derived solutions. The preliminary results enhance the contribution of Galileo system and pave also the way to a wider use of lowcost single frequency receivers for specific monitoring applications. The state of the undergoing development of the VADASE software for the combined processing of GPS and Galileo data are also presented.

Keywords: variometric approach; displacement analysis; Galileo observations

\section{Introduction}

Since the mid-90s, thanks to technological developments of GPS receivers, it has been possible to acquire GPS observations at high frequencies $(1 \mathrm{~Hz}$ or above). Beside other new applications, GPS technology was used since then to estimate displacements and waveforms due to earthquakes. Significant contributions have been provided by several authors (e.g., Bock et al., 2000; Langbein and Bock, 2004; Blewitt et al., 2006; Bock and Genrich, 2006). In this field, the main advantage over seismometers is the non saturation of GPS derived solutions (Larson, 2009). In the years, two approaches emerged in GPS seismology, namely: differential positing (DP) (Ohta et al., 2012) and precise point positioning (PPP) (Bock et al., 1993; Kouba, 2003; Larson et al., 2007; Larson, 2009; Xu et al., 2012; Hung and Rau, 2013). Commonly, the two approaches require dual frequency observations and, in general, ambiguity resolution. The first approach relies on permanent stations networks and information technology (IT) infrastructures; the second requires precise products. Advances made in recent years are being opening its use in real-time warning systems, e.g., the Real-time Earthquake Analysis for Disaster Mitigation Network ${ }^{1}$. More recently, the development of the variometric approach for displacement analysis and its successful applications of its VADASE (Variometric Displacement Analysis Approach for Stan-Alone Engine) implementation emerged as a third option in GPS seismology (Colosimo et al., 2011). This approach relies on high-frequency observations from a stand-alone receiver and broadcast products only; there is no need of infrastructures and real-time applications are straightforward.

\footnotetext{
${ }^{1}$ http://sopac-csrc.ucsd.edu/index.php/readi
} 
On December 2016 the Galileo system started to offer its initial services to public users.

To support the adoption of Galileo data for critical and real-time applications of the variometric approach, in this work high-rate observations acquired at the time of the European Commission declaration of the start of the Galileo Initial Services were investigated. A late 2017 campaign was also considered for evaluating the impact of the evolution of the Galileo system so far. A comparison between solutions based on GPS only and Galileo only dataset was carried out. A combined single frequency dataset was also considered in a simplified processing. Single frequency observations were also processed using the same simplified version of the full dual frequency model. This opens the way to applications in application fields where low-cost single frequency receivers are preferable to geodetic ones.

In the following section, the variometric model for displacement analysis is briefly recalled. It follows a description of dataset and of the procedure developed for the evaluation of the Galileo based solutions of the variometric model. Results are then presented and discussed; eventually future prospects are proposed.

\section{The variometric approach for displacement analysis}

The VADASE implementation provides the estimation of epoch-by-epoch displacements by solving a system of time single-difference of observations collected at two consecutive epochs. A set of at least four epoch-to-epoch common satellites is required. Cycle slips must be identified and removed; phase observations are processed without the need of ambiguity resolution. The observation equation of the variometric model reads:

$$
\underbrace{\alpha \lambda_{1} \Delta \Phi_{1}+\beta \lambda_{2} \Delta \Phi_{2}}_{\begin{array}{c}
\text { time single-differences of } \\
\text { iono-free observations }
\end{array}}=\underbrace{\left(\nu \cdot \Delta \xi_{r}+c \Delta \delta t_{r}\right)}_{\text {unknown terms }}+\underbrace{\Delta \rho-c \Delta \delta t^{s}+\Delta T+\Delta p}_{\text {known terms }}+\underbrace{\Delta m+\Delta \epsilon}_{\text {noise }}
$$

where $\Delta \Phi_{i}$ is the carrier phase observation time difference between two consecutive epochs and $\lambda_{i}$ is the carrier phase wavelength, with $i=1,2$ for the first and second carrier phase respectively; the coefficients of the iono-free

combination are $\alpha$ and $\beta$; the subscript $r$ and the superscript $s$ are the com- 
mon receiver and satellite identification indexes ${ }^{2} ; \nu$ is the satellite-receiver unit vector; $\Delta \xi_{r}$ is the receiver displacement in the epoch-to-epoch interval; $c$ is the speed of light; $\delta t$ is the clock error; $T$ is the tropospheric delay; $p$ includes model contributions for relativistic effects, phase center variations, phase windup effect; $m+\epsilon$ are multipath and noise contributions. The change of the geometric distance $\Delta \rho$ accounts for satellite's orbital motion, Earth's rotation, solid Earth tides and ocean loading phenomena. In Eq. (1) the unknowns are the epoch-to-epoch receiver clock error variation and epochto-epoch antenna displacement, which is equivalent to a velocity. Known terms are computed applying appropriate models.

After a proper simplification, the variometric model can also be applied using single frequency observations only. For a given carrier phase $i$, the simplified model now reads:

$$
\lambda_{i} \Delta \Phi_{i}=\left(\nu \cdot \Delta \xi_{r}+c \Delta \delta t_{r}\right)+\Delta \rho_{O R}-c \Delta \delta t^{s}+\Delta T+\Delta I+\Delta m+\Delta \epsilon
$$

where tropospheric and ionospheric delays, $\Delta T$ and $\Delta I$ respectively, are computed by means of Saastamoinen (1972) and Klobuchar (1987) models; relativistic effects are omitted for the seek of compactness; phase center variations and phase windup effect are neglected. The change of the geometric distance $\Delta \rho_{O R}$ accounts only for satellite's orbital motion and Earth's rotation. Eqs. (1) and (2) can be used also for code observations after standard substitution of observed quantities.

Various applications of VADASE have proved the effectiveness of the approach in providing three dimensional displacements of a fast moving receiver with high accuracy in a global reference frame (Branzanti et al., 2013; Benedetti et al., 2014).

\section{Data selection and preparatory processing}

At the time of the Declaration of the Initial Service of the Galileo system (December 2016) only eleven Galileo satellites were operational. A special procedure was therefore developed in order to identify satellite configurations of the two systems that were, according to specific criteria, as close as

\footnotetext{
${ }^{2}$ Receiver and satellite indexes are omitted for the seek of simplicity where no ambiguity is at risk.
} 
possible, thus leading to fair comparisons between GPS based results and Galileo based ones. A set of 32 IGS Multi-GNSS (MGEX) stations spread worldwide were considered. A number greater or equal to three stations were selected within each of four classes of latitude to build a station dataset for a reasonable representation of different conditions of satellite visibility. Figure 1 shows a map view of the 32 stations and the amplitudes of the four classes [Cls \#1-4] of latitude considered in this study.

[Figure 1 about here.]

Observation sessions lasting 10 days (2-12 December 2016) were selected in order to consider all possible satellite configurations over a full repeat period of the Galileo system. For the same time period, observations from a low-cost L1 GNSS receiver located in Trento, Italy, were also collected. Every observation session was split in 15 min sub-sessions. For every sub-session, the Position Dilution of Precision (PDOP) index and the overall number of satellites in view were computed. For every sub-session, observations at 30 s rate were processed using VADASE to estimate a sub-session specific solution noise. Sub-sessions were then ranked according to the values of the above given quantities to select the 15 min sub-section per station with the best geometry, highest number of satellites in view and lowest solution noise. In particular only sub-sessions presenting a PDOP value lower than 1.6 and with at least 6 satellites were selected, resulting on a set of 31 time windows for just 12 of the 32 stations considered. The criteria adopted for the identification of the sub-sessions to be further processed were aimed at selecting datasets suitable to better highlight just the impact of the different satellite observations on VADASE solutions and hence affected as little as possible from other sources of uncertainty.

The number of visible satellites and PDOP affected the amplitude of the fluctuations. As an example, East component of the velocity of the ANMG site estimated form VADASE processing of $30 \mathrm{~s}$ code observations is plotted in Figure 2 along with PDOP and number of satellites in view versus GPS seconds-of-week [SOW]. Figure 3 shows boxplots ${ }^{3}$ of the displacements in East, North, Up components from VADASE processing of 10 days of $30 \mathrm{~s}$

\footnotetext{
${ }^{3}$ The first and third quartiles of the dataset are the bottom and top lines of the box, the second quartile (the median) is the line inside the box, the ends of the whiskers represent the minimum and the maximum values respectively.
} 
iono-free phase observations (E1 \& E5a) and for all the 32 MGEX stations (Year 2016). During the studied time period, stations were not affected by displacements thus reference velocity value was set equal to zero and solution fluctuations interpreted as solution noise only. With respect to the expected zero reference displacement, differences of the displacements mean values of the 32 stations here considered were in the order of $1 \mathrm{~mm}$; root-mean-square errors (RMSE) of every dataset of station solutions were in the decimeter order. Figure 4 shows RMSE of the selected 31 sub-sessions for different satellite configurations, with respect to PDOP and number of satellites.

[Figure 2 about here.]

[Figure 3 about here.]

[Figure 4 about here.]

For every latitude class the station with the overall best sub-session was eventually selected for further processing. The MGEX stations selected were: CHPG, CPVG, RGDG and YEL2. According to the same criteria, the best sub-section was selected also for the site named Mesi in Trento. The five selected sub-sessions were later processed using VADASE and phase observations at $1 \mathrm{~s}$ rate (see Section 4 ).

The same procedure was adopted for the selection of two new 15 min subsections in data acquired at the YEL2 and Mesi stations in December 2017, one year after the acquisition period of the initial dataset considered before. In December 2017, 15 Galileo satellites were operational.

For the number of operational GPS satellites being greater than the number of operational Galileo satellites, geometrically similar satellite configurations were selected to properly compare GPS and Galileo solutions both in 2016 and 2017. To this end, two algorithms were developed to identify the GPS satellites to be removed in order to obtain an equal configuration in terms of number of satellites and a similar configuration in terms of geometry. This second goal was achieved through two distinct geometric likelihood criteria. The first one (criterion 1) maximizes the sum of the scalar product of the $\nu$ unit vectors, the second one (criterion 2) minimizes the PDOP difference of the two configurations. Figure 5 shows skyplots of Galileo and complete GPS satellite configurations on the left; skyplots on the right show the two GPS configurations reduced according to the described criteria. Highlighted areas are the base of the pyramids the volume of which determines the PDOP values. 
[Figure 5 about here.]

\section{Results of phase observations processing}

To investigate accuracy and precision of VADASE solutions in a standard application setup, a further processing of $1 \mathrm{~s}$ rate phase observations of the sub-sections previously selected was conducted. Th model was tested with both single frequency observations (E1, E5, L1) and iono-free phase observations (E1 \& E5a and L1 \& L2). The solutions were obtained using both Galileo and GPS observations individually, then compared. To evaluate the impact of E1-L1 interoperability a combined GPS\&Galileo solution was investigated. To this end the functional model, Eq. (2), was properly simplified. In the simplified model tropospheric and ionospheric delays were computed by Saastamoinen and Klobuchar models for both systems and a single $\Delta \delta t_{r}$ unknown was estimated for both systems assuming that, for short time intervals, receiver clock errors are the same in both the time scales of the GPS and Galileo systems. The combined solutions were computed by stacking all GPS and Galileo observation equations.

VADASE solutions obtained by processing single frequency observations acquired by RGDG station are shown in Figure 6. All solutions present RMSE at the millimeter level. The combined solution presents the better results, the GPS solution with full constellation is slightly better than the Galileo solution. With respect to the Galileo solution, the GPS solutions with reduced constellations present comparable RMSE when the algorithm that minimizes the PDOP is applied and slightly higher RMSE when the algorithm that maximizes the geometric similitude is applied. As a whole, RMSE obtained from the processing of Galileo single frequency observations form 4 MGEX stations and u-blox receiver in Trento (Mesi) are within the $(2,4) \mathrm{mm} / \mathrm{s}$ range for the East and North components and lower than 5 $\mathrm{mm} / \mathrm{s}$ for the Up component. The use of GPS observations results in similar RMSE for the East and North components and in RMSE lower than $8 \mathrm{~mm} / \mathrm{s}$ for the Up component. The combined use of the two systems is characterized by RMSE of nearly $1 \mathrm{~mm} / \mathrm{s}$ for the East and North components and RMSE of about $3 \mathrm{~mm} / \mathrm{s}$ for the Up component. In particular, for the accuracy of the YEL2 station solutions is of a few millimeter only $\left(0.9 \mathrm{~mm} / \mathrm{s} \mathrm{V}_{\text {East }}, 1.3\right.$ $\left.\mathrm{mm} / \mathrm{s} \mathrm{V}_{\text {North }}, 2.7 \mathrm{~mm} / \mathrm{s} \mathrm{V}_{\mathrm{Up}}\right)$. For the Mesi site in Trento solutions accuracy is only slightly lower $\left(2.6 \mathrm{~mm} / \mathrm{s} \mathrm{V}_{\text {East }}, 1.2 \mathrm{~mm} / \mathrm{s}_{\text {North }}, 3.1 \mathrm{~mm} / \mathrm{s} \mathrm{V}_{\mathrm{Up}}\right)$.

[Figure 6 about here.] 
Figure 7 shows the VADASE results for single frequency observations collected in 2017 at the YEL2 site. During the selected 15 min sub-session within days 310-319, there were 9 Galileo satellites in view. Figure 8 shows the VADASE results for single frequency observations collected in 2017 at the Mesi site in Trento. During the selected 15 min sub-session there were 8 Galileo satellites in view. Quality of VADASE solutions based on single frequency observations collected in 2017 at the YEL2 and Mesi sites are summarized in Table 1.

[Figure 7 about here.]

[Figure 8 about here.]

Table 1: Quality summary of VADASE solutions based on single frequency observations; YEL2 and Mesi sites (Year 2017).

\begin{tabular}{llllr} 
& & \multicolumn{3}{c}{ RMSE $[\mathrm{mm} / \mathrm{s}]$} \\
\hline \multirow{4}{*}{ YEL2 } & & E1 & L1 & E1\&L1 \\
\cline { 3 - 5 } & $\mathrm{V}_{\text {East,North }}$ & 0.7 & 1.3 & 0.7 \\
& $\mathrm{~V}_{\text {Up }}$ & 1.5 & 1.8 & 1.4 \\
\hline \multirow{2}{*}{ Mesi } & $\mathrm{V}_{\text {East,North }}$ & 1.9 & 2.6 & 1.8 \\
& $\mathrm{~V}_{\text {Up }}$ & 2.9 & 4.0 & 2.9 \\
\hline
\end{tabular}

Results for dual frequency observations from 4 MGEX stations are not significantly better than the results reported for single frequency observations. Sensitivity of the L2 carrier balances the removal of the ionospheric term, leading to comparable results between single frequency and dual frequency solutions. Quality of VADASE solutions based on dual frequency observations acquired by geodetic receivers is shown in Figure 9 and summarized in Table 2.

[Figure 9 about here.]

In this work observations collected from GNSS stations not affected by earthquake-like displacements were considered. To evaluate the impact of Galileo observations on the solutions of the variometric approach a sort of zero-displacement scenario was considered and the analysis of the results was 
Table 2: Quality summary of VADASE solutions based on iono-free phase observations; MGEX sites (Year 2017).

\begin{tabular}{lrrr}
\multicolumn{4}{c}{ RMSE ranges [mm/s] } \\
\hline & E1 \& E5a & $\begin{array}{r}\text { L1 \& L2 } \\
\text { [full conf. }]\end{array}$ & $\begin{array}{r}\text { L1 \& L2 } \\
\text { [reduced conf.] }\end{array}$ \\
\cline { 2 - 4 } $\mathrm{V}_{\text {East,North }}$ & $(2,3)$ & $(2,3)$ & $(2,3)$ \\
$\mathrm{V}_{\mathrm{Up}}$ & $(4,5)$ & $(4,7)$ & $(4,8)$ \\
\hline
\end{tabular}

carried out essentially in the velocity domain. The integration of the velocities over short period is however illustrative even in this scenario. Figure 10 shows the displacements obtained form Galileo only data, GPS from only data, and from the combined Galileo\&GPS data, respectively. A period of 4 min was considered for such a time interval would be wide enough to permit the description even of catastrophic seismic events. The plots highlight the smoothness of the Galileo solutions with respect to the GPS solutions. In the plots, drifts due to integration of noisy velocities are clearly visible. Anyway, it has been demonstrated that for short periods, e.g. during real seismic event, by means of the variometric approach it is possible to retrieve waveforms and co-seismic displacements in real time (Fratarcangeli et al., 2018).

[Figure 10 about here.]

\section{Discussions and Future prospects}

In this work, the variometric model for displacement analysis was applied to Galileo observations providing solutions of comparable or better quality than those obtained using GPS observations only. The quality of Galileo solutions is comparable or better than GPS, even when considering a number of Galileo satellites smaller than the number of GPS satellites. In general, results show that combined GPS\&Galileo solutions are better than singlesystem ones. The processing of single frequency observations presented in this work concurred to provide a wide overview about the impact of the Galileo observations on the quality of VADASE solutions. The quality of solutions based on single frequency observations is comparable to or even of better quality than the quality of solutions based on dual frequency observations. This can be in part due to the influence on the iono-free combination of the 
lower signal-to-noise level of L2 frequency with respect to L1. Moreover, for time periods of a few minutes, the use of standard models to describe troposphere and ionosphere delays, and the use of broadcast orbits do not compromise the quality of VADASE solutions. The remarkable precisions and accuracies reached using single frequency observations support the possibility of using low-cost receivers for specific purposes. Interoperability foreshadows important developments in the field of GNSS seismology and in other fields motivating the need of further investigations on the performances of multiconstellations receivers.

The quality of results based on the use of observations acquired in December 2017, one year after the start of Galileo Initial Services operational phase, is improved for the greater satellite availability. Completion of new systems and modernization of existing ones are expected to improve VADASE performance even more.

Further developments include the widening of test and applications of the variometric model, in particular in real-time scenarios with real displacements. The combined GPS\&Galileo variometric model can be improved in order to consider one receiver clock parameter per system. Interoperability capabilities can be expanded in order to deal with observations from multiple constellations including, e.g., Beidou and QZSS.

\section{References}

Benedetti, E., , Branzanti, M., Biagi, L., Colosimo, G., Mazzoni, A., Crespi, M., 2014. Global navigation satellite systems seismology for the $2012 \mathrm{Mw}$ 6.1 emilia earthquake: Exploiting the vadase algorithm. Seismological Research Letters 85 (3), 649-656, doi: 10.1785/0220130094.

Blewitt, G., Kreemer, C., Hammond, W., Plag, HP. and, S. S., Okal, E., 2006. Rapid determination of earthquake magnitude using GPS for tsunami warning systems. Geophys Res Lett 33 (11), L11309, doi: 10.1029/2006GL026145.

Bock, Y., Agnew, D., Fang, P., et al., 1993. Detection of crustal deformation from the landers earthquake sequence using continuous geodetic measurements. Nature 361 (6410), 337-340, doi: 10.1038/361337a0.

Bock, Y., Genrich, J., 2006. Instantaneous geodetic positioning with 10-50 Hz 
GPS measurements: noise characteristics and implications for monitoring networks. J Geophys Res 111 (3), B03403, doi: 10.1029/2005JB003617.

Bock, Y., Nikolaidis, R., de Jonge, P., Bevis, M., 2000. Instantaneous geodetic positioning at medium distances with the global positioning system. J Geophys Res 105 (B12), 28223-28253, doi: 10.1029/2000JB900268.

Branzanti, M., Colosimo, G., Crespi, M., Mazzoni, A., 2013. GPS near-real-time coseismic displacements for the great Tohoku-oki earthquake. IEEE Geosci Remote Sens Lett 10 (2), 372-376, doi: 10.1109/LGRS.2012.2207704.

Colosimo, G., Crespi, M., Mazzoni, A., 2011. Real-time GPS seismology with a stand-alone receiver: a preliminary feasibility demonstration. J Geophys Res 116 (11), B11302, doi: 10.1029/2010JB007941.

Fratarcangeli, F., Ravanelli, M., Mazzoni, A., Colosimo, G., Benedetti, E., Branzanti, M., Savastano, G., Verkhoglyadova, O., Komjathy, A., Crespi, M., 2018. The variometric approach to real-time high-frequency geodesy. Rendiconti Lincei. Scienze Fisiche e Naturali 29 (S1), 95-108, doi $=10.1007 / \mathrm{s} 12210-018-0708-5$.

Hung, H., Rau, R., 2013. Surface waves of the 2011 Tohoku earthquake: observations of Taiwans dense high-rate GPS network. J Geophys Res 118 (1), 332-345, doi: 10.1029/2012JB009689.

Klobuchar, J., 1987. Ionospheric time-delay algorithmn for single-frequency GPS users. IEEE Trans Aerosp Electron Syst AES-23 (3), 325-331, doi: 10.1109/TAES.1987.310829.

Kouba, J., 2003. Measuring seismic waves induced by large earthquakes with GPS. Stu Geophys et Geod 47 (4), 741-755, doi: 10.1023/A:1026390618355.

Langbein, J., Bock, Y., 2004. High-rate real-time GPS network at parkfield: Utility for detecting fault slip and seismic displacements. Geophys Res Lett 31 (15), L15S20, doi: 10.1029/2003GL019408.

Larson, K., 2009. GPS seismology. J Geod 83 (3-4), 227233, doi: 10.1007/s00190-008-0233-x. 
Larson, K., Bilich, A., Axelrad, P., 2007. Improving the precision of high-rate GPS. J Geophys Res 112 (5), B05422, doi: 10.1029/2006JB004367.

Ohta, Y., Kobayashi, T., Tsushima, H., Miura, S., Hino, R., Takasu, T., Fujimoto, H., Iinuma, T., Tachibana, K., Demachi, T., Sato, T., Ohzono, M., Umino, N., 2012. Quasi real-time fault model estimation for near-field tsunami forecasting based on RTK-GPS analysis: application to the 2011 Tohoku-oki earthquake (Mw 9.0). J Geophys Res 117 (2), B02311, doi: 10.1029/2011JB008750.

Saastamoinen, J., 1972. Atmospheric correction for the troposphere and stratosphere in radio ranging of satellites and in The Use of Artificial Satellites for Geodesy. Geophys Monogr Ser 15, 247-251, doi: 10.1029/GM015p0247.

Xu, P., Shi, C., Fang, R., Liu, J., Niu, X., Zhang, Q., Yanagidani, T., 2012. High-rate Precise Point Positioning (PPP) to measure seismic wave motions: an experimental comparison of GPS PPP with inertial measurement units. J Geod 87 (4), 361-372, doi: 10.1007/s00190-012-0606-z. 


\section{List of Figures}

1 World map with the 32 stations and the amplitudes of the four classes [Cls \#1-4] of latitude considered in this study . . . . . 14

2 Velocity in East component from VADASE processing of $30 \mathrm{~s}$ code observations (ANMG site), PDOP, and number of satellites versus GPS seconds-of-week (Year 2016). . . . . . . . . . 15

3 Statistics of displacements in East (E), North (N) and Up (U) components from VADASE processing of 10 days of $30 \mathrm{~s}$ ionofree phase observations (E1 \& E5a) for 32 MGEX stations (Year 2016) . . . . . . . . . . . . . . . . . . 16

4 Velocity RMSE of 15 min sub-sessions for different satellite configurations, with respect to PDOP and number of satellites (Year 2016) . . . . . . . . . . . . . . . . . . 17

5 Skyplots of: a) Galileo and complete GPS satellite configurations and b) reduced GPS satellite configurations (Year 2016). 18

6 VADASE solutions based on single frequency observations: a) Galileo E1, b) GPS L1, c) Galileo E1 + GPS L1, d) and e) GPS L1 with reduced satellite configurations; RGDG site (Year 2017). 19

$7 \quad$ VADASE solutions based on single frequency observations: a) Galileo E1, b) GPS L1, c) Galileo E1 + GPS L1; YEL2 site (Year 2017) . . . . . . . . . . . . . . . . . . . 20

8 VADASE solutions based on singe frequency observations: a) Galileo E1, b) GPS L1, c) Galileo E1 + GPS L1; Mesi site (Year 2017) . . . . . . . . . . . . . . . . . . . . . . 21

9 Quality summary of VADASE solutions based on iono-free phase observations: a) Galileo E1 \& E5, b) GPS L1 \& L2, c) and d) GPS L1 \& L2 with reduced satellite configurations; MGEX sites (Year 2016). . . . . . . . . . . . . . . . . . 22

10 Example of displacement components based on: a) Galileo data, b) GPS data, c) combined Galileo\&GPS ; YEL2 site (Year 2017) . . . . . . . . . . . . . . . . . . 23 


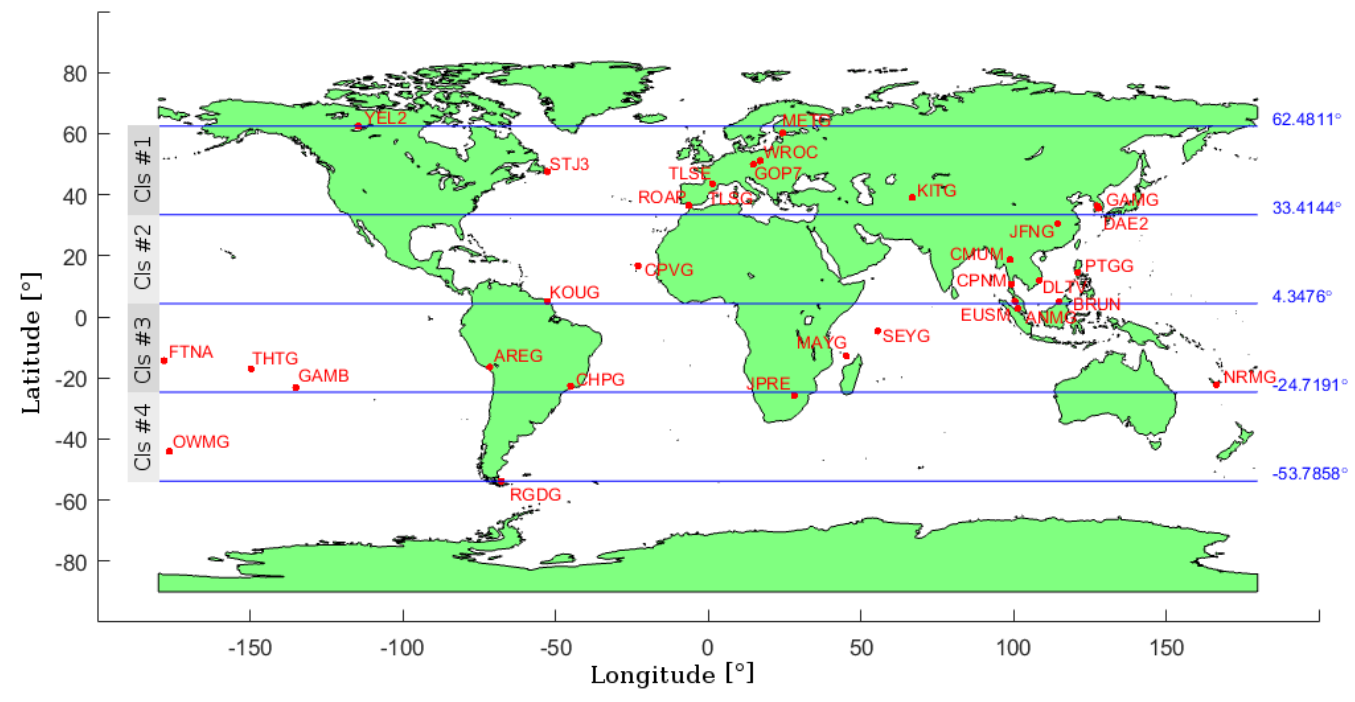

Figure 1: World map with the 32 stations and the amplitudes of the four classes [Cls \#1-4] of latitude considered in this study 


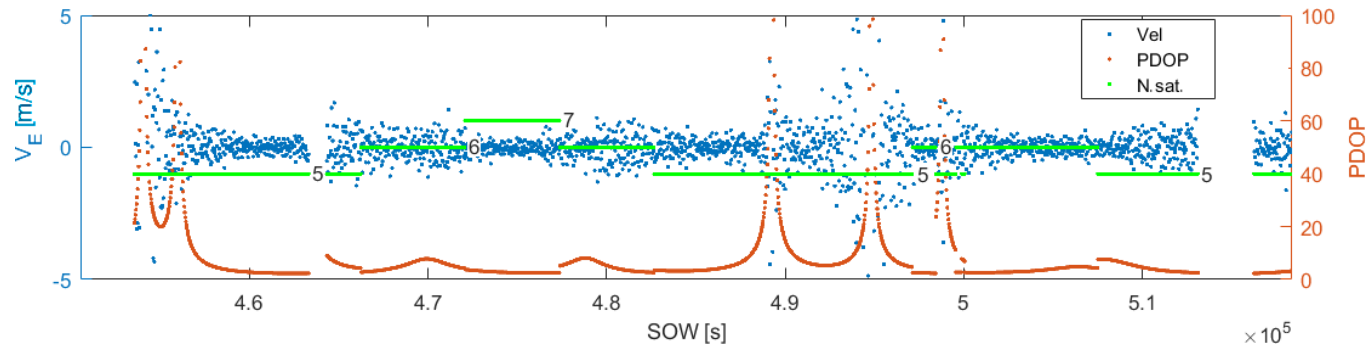

Figure 2: Velocity in East component from VADASE processing of $30 \mathrm{~s}$ code observations (ANMG site), PDOP, and number of satellites versus GPS seconds-of-week (Year 2016). 

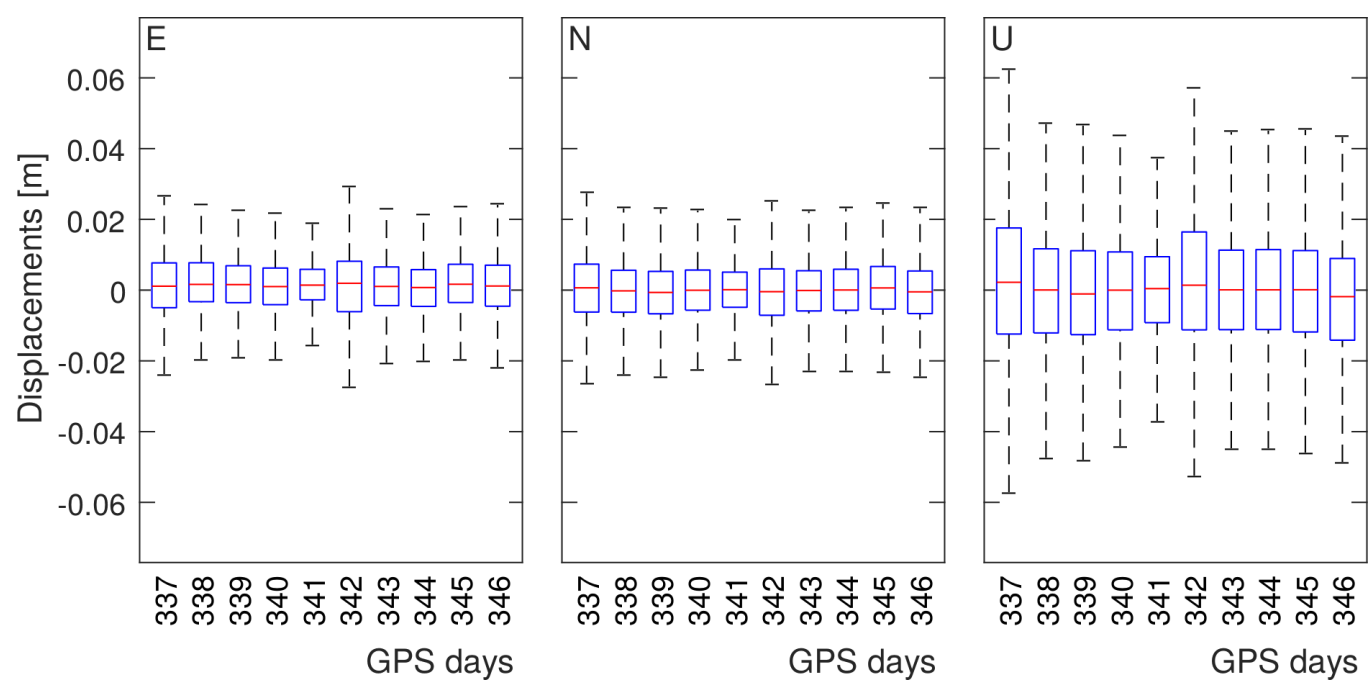

Figure 3: Statistics of displacements in East (E), North (N) and Up (U) components from VADASE processing of 10 days of $30 \mathrm{~s}$ iono-free phase observations (E1 \& E5a) for 32 MGEX stations (Year 2016). 


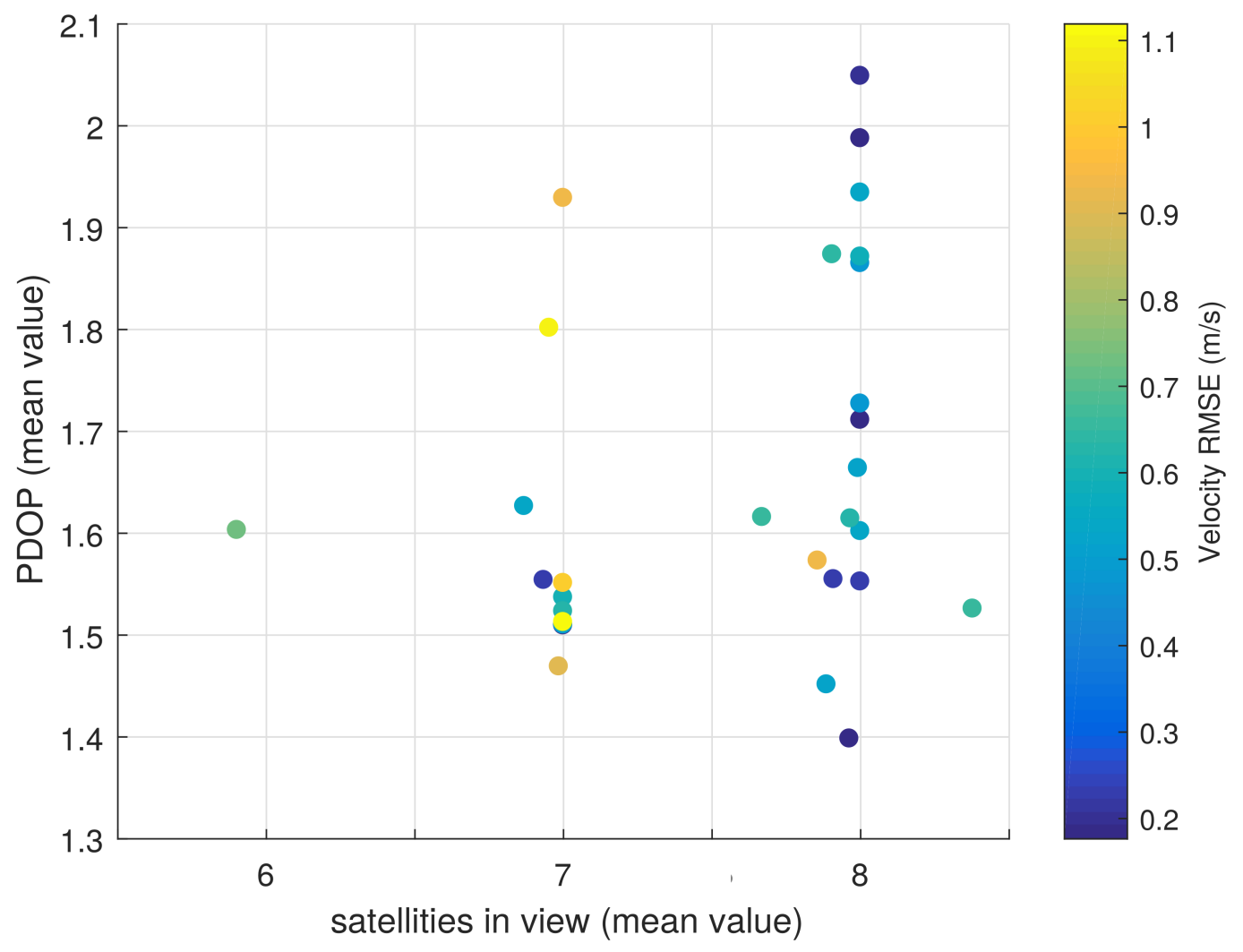

Figure 4: Velocity RMSE of 15 min sub-sessions for different satellite configurations, with respect to PDOP and number of satellites (Year 2016). 


\section{RDGD station}

GPS day 339, 23:45 - 00:00

\begin{tabular}{l|c|c} 
& Galileo & GPS \\
\hline $\mathrm{N}^{\circ}$ satelites & 8 & $11(13)$ \\
$\mathrm{PDOP}$ & 1.73 & 1.60
\end{tabular}

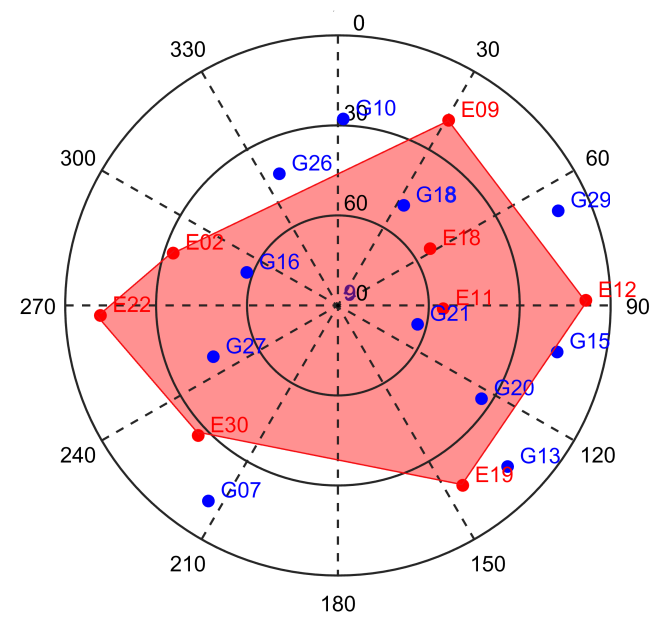

\section{Ei Galileo satelites Gi GPS satelites}

a)

\section{First algorithm PDOP: 2.73}

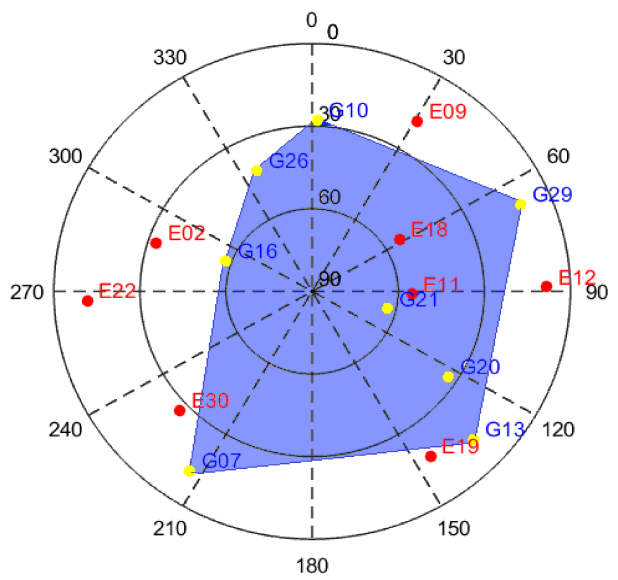

Second algorithm PDOP: 1.73

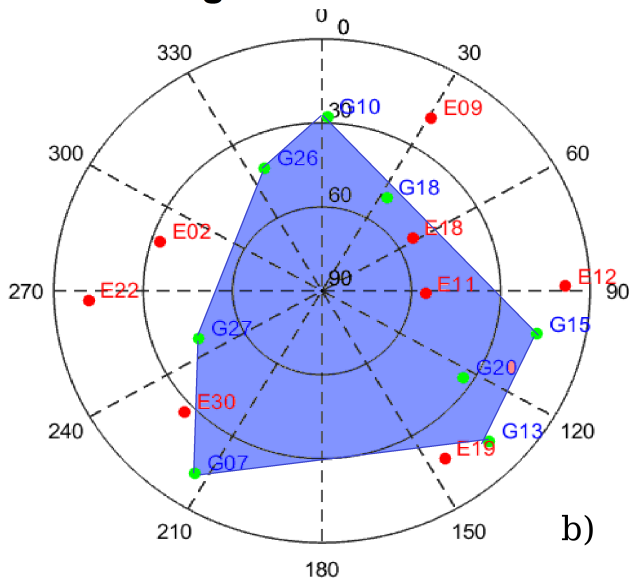

Figure 5: Skyplots of: a) Galileo and complete GPS satellite configurations and b) reduced GPS satellite configurations (Year 2016). 


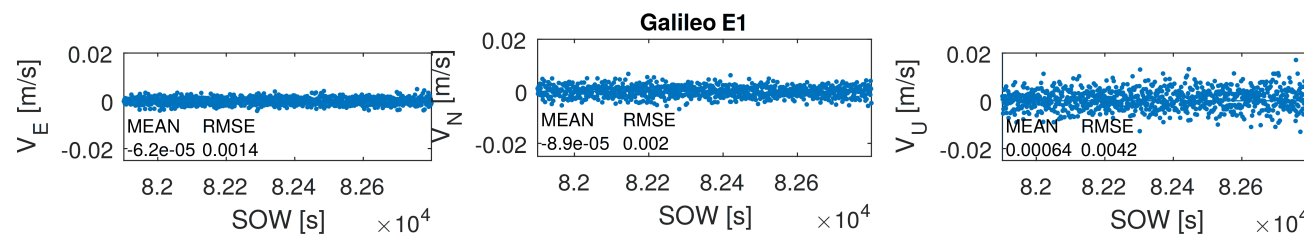

a)
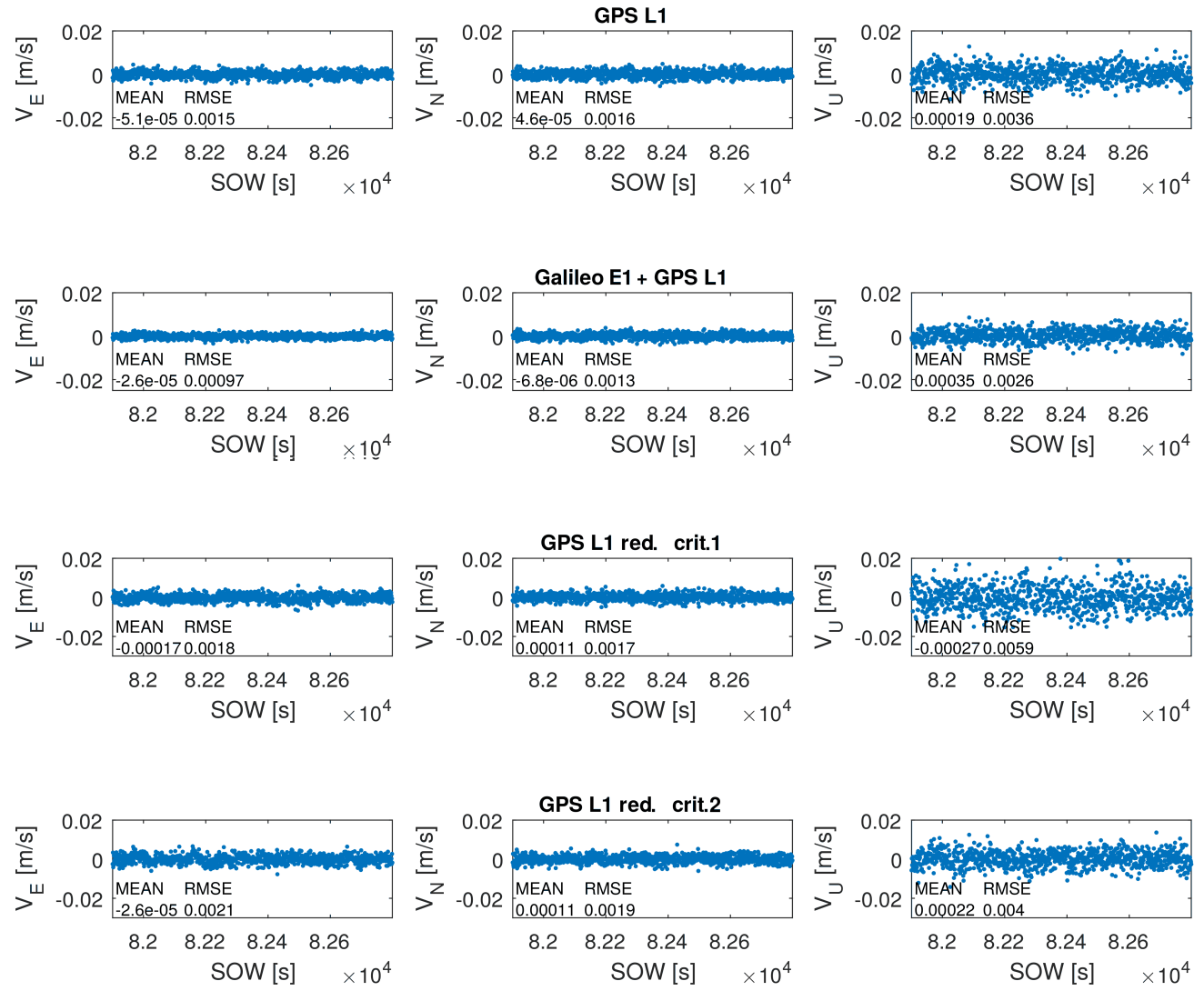

Figure 6: VADASE solutions based on single frequency observations: a) Galileo E1, b) GPS L1, c) Galileo E1 + GPS L1, d) and e) GPS L1 with reduced satellite configurations; RGDG site (Year 2017). 

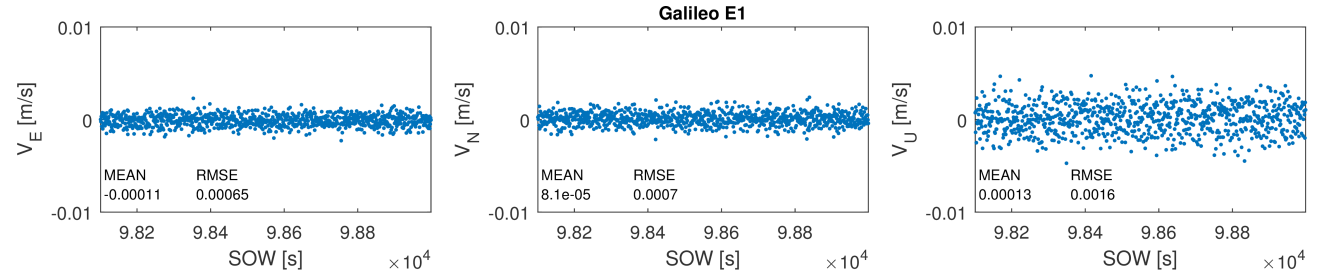

a)
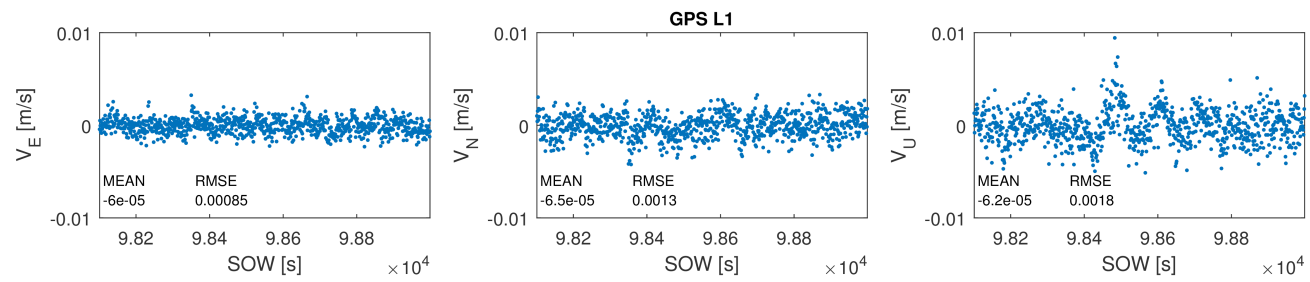

b)
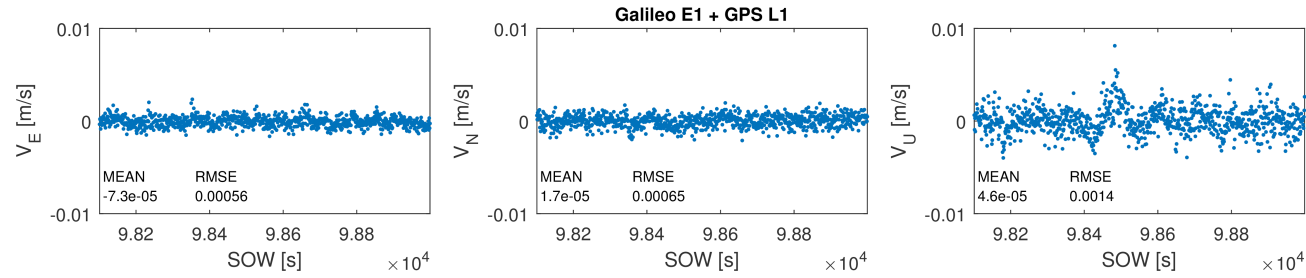

Figure 7: VADASE solutions based on single frequency observations: a) Galileo E1, b) GPS L1, c) Galileo E1 + GPS L1; YEL2 site (Year 2017). 

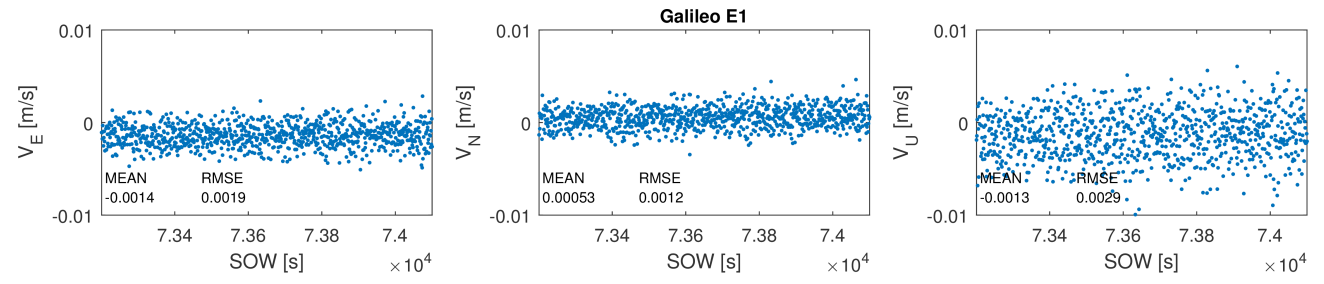

a)
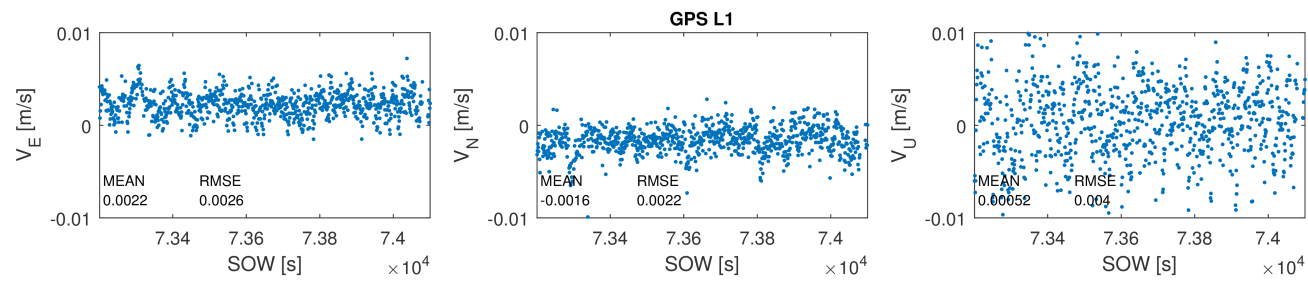

b)
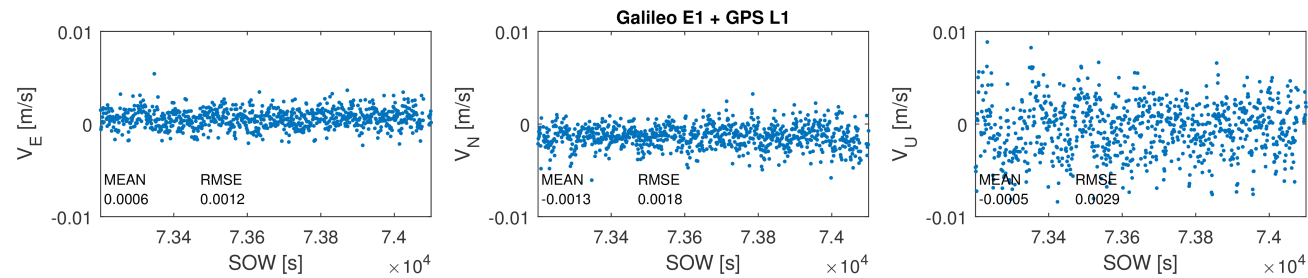

C)

Figure 8: VADASE solutions based on singe frequency observations: a) Galileo E1, b) GPS L1, c) Galileo E1 + GPS L1; Mesi site (Year 2017). 


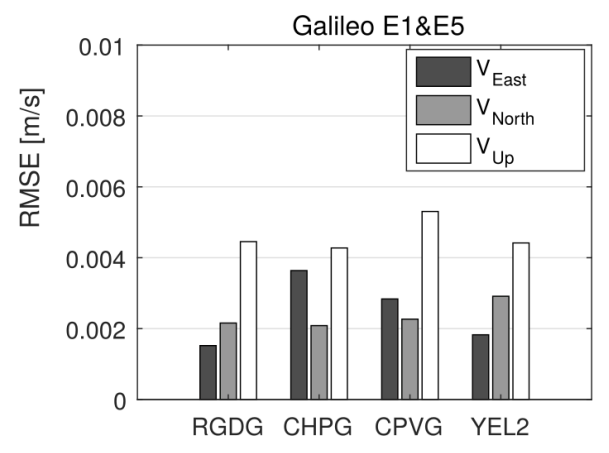

a)

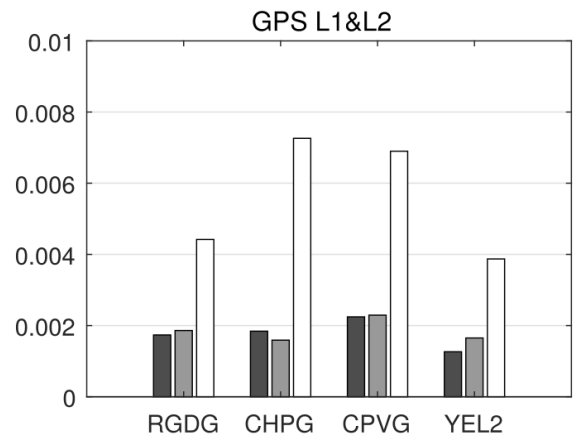

b)
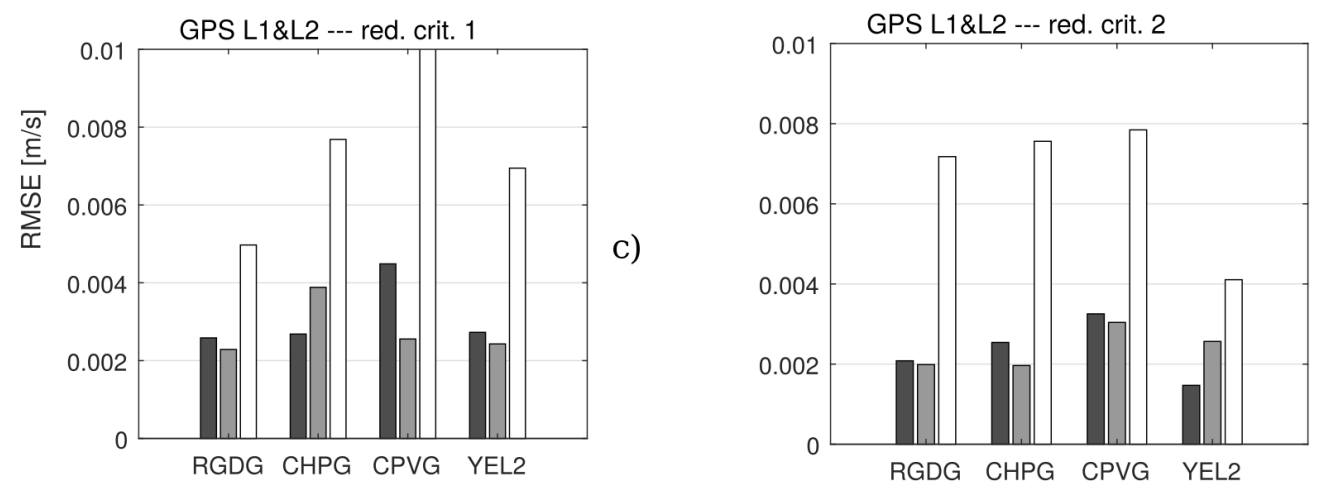

d)

Figure 9: Quality summary of VADASE solutions based on iono-free phase observations: a) Galileo E1 \& E5, b) GPS L1 \& L2, c) and d) GPS L1 \& L2 with reduced satellite configurations; MGEX sites (Year 2016). 


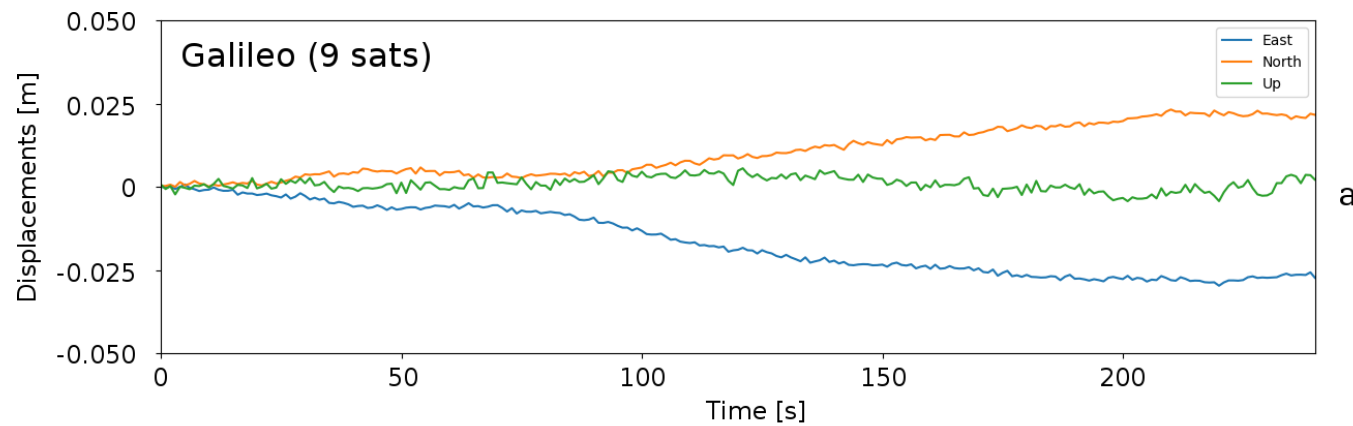

a)

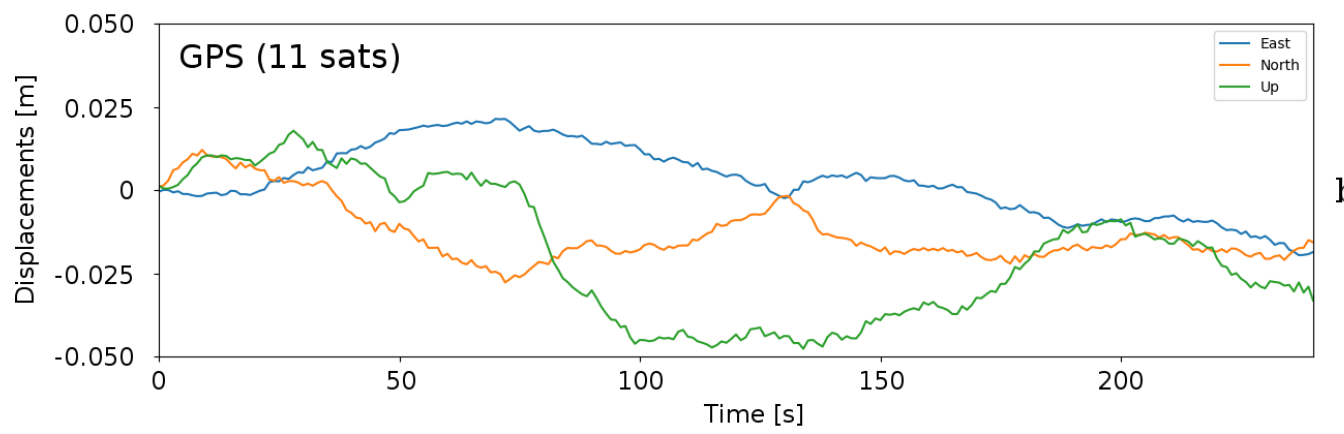

b)

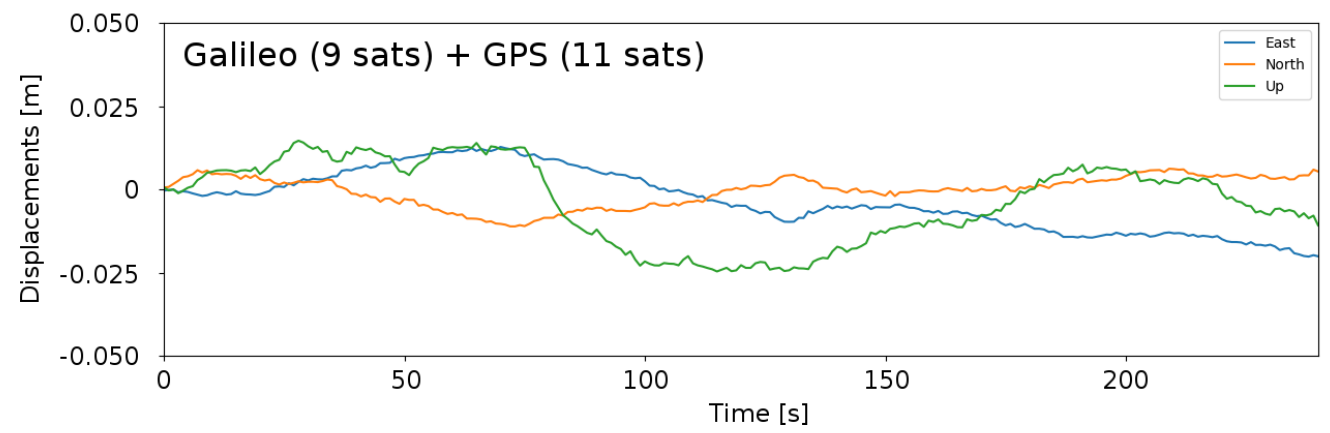

c)

Figure 10: Example of displacement components based on: a) Galileo data , b) GPS data, c) combined Galileo\&GPS ; YEL2 site (Year 2017). 